\title{
The Effect of $\boldsymbol{N}$-Alkyl Substituents on the Usability of Imidazolium Cation-Based Ionic Liquids in Microemulsion Systems: A Technical Note
}

\author{
Neeraj Kumar, ${ }^{1}$ Shishu Goindi, ${ }^{1,3}$ Sandeep Kumar, ${ }^{2}$ and Asim Kumar Jana ${ }^{2}$
}

Received 6 November 2012; accepted 14 February 2013; published online 6 March 2013

KEY WORDS: drug delivery; hemolysis; imidazolium; ionic liquids; microemulsion.

\section{INTRODUCTION}

Ionic liquids (ILs) are predominantly hailed as green solvents, due to their non-volatile, recyclable and non-hazardous nature. Their wide-spectrum applications can be implemented into conventional fields like lubricants, battery electrolytes and synthetic reaction media as well as newer arenas including supramolecular chemistry, separation techniques, and nuclear fuel reprocessing (1-4).

However, due to their tunable physicochemical properties, some recent reports have suggested novel pharmaceutical uses of ionic liquids, including their use as active pharmaceutical ingredient salts (5), antimicrobials (6), solubilizers (7), and drug delivery systems $(8,9)$.

ILs are known to enhance the solubility profile of poorly water-soluble drugs $(7,10)$. Therefore, the use of IL-based drug carrier systems could be a rational approach for delivery of insoluble drugs.

Microemulsions are thermodynamically stable, isotropic systems composed of a polar phase (usually water), a nonpolar phase (oils) and amphiphilic phase (surfactant or mixture of surfactants). These systems provide numerous advantages over conventional drug delivery vehicles including nanometer sized aggregations, long-term stability, biocompatibility, easy preparation, and high solubilization tendency (11). Nevertheless, drug delivery using microemulsions is yet to fully attain its true potential, considering that only type and content of oils and surfactants have been customized, in general, by formulators to achieve different desirable characteristics. Little efforts have been performed in the direction of modifying the polar phase of microemulsion systems. We

Electronic supplementary material The online version of this article (doi:10.1208/s12249-013-9939-z) contains supplementary material, which is available to authorized users.

\footnotetext{
${ }^{1}$ University Institute of Pharmaceutical Sciences (U.I.P.S.), UGC Centre of Advanced Study, Panjab University, Chandigarh, 160014, India.

${ }^{2}$ Department of Biotechnology, Dr. B. R. Ambedkar National Institute of Technology, Jalandhar, 144011, Punjab, India.

${ }^{3}$ To whom correspondence should be addressed. (e-mail: shishugoindi@yahoo.co.in)
}

attempted to use ILs as alternative polar dispersed phase (in place of water) in the microemulsion systems. Moniruzzaman et al., recently have prepared the IL-based microemulsion carrier for drug delivery of poorly hydrophilic drugs (12). The report describes the use of dimethylimidazolium dimethylphosphate for successful transdermal delivery of poorly soluble drugs. However, to the best of our knowledge, we are for the first time reporting the screening of room temperature ionic liquids from two different series of 1- and 3-alkyl substituted imidazolium bromides to be used as components of microemulsion vehicles.

The current study explains the synthesis of some imidazolium cation-based ILs with varying carbon chain length. Also, preliminary in vivo toxicity of these compounds was assessed by hemolysis assay using human blood to establish the possibility of using the ILs safely, and investigating relationship between $N$-alkyl substituents and their toxicity. Further, their effect on the microemulsion phase behavior was studied by constructing their pseudoternary phase diagrams using isopropyl myristate (IPM) as oil phase and Span 80 and Tween 80 mixture as emulgent phase. Model microemulsion systems consisting of the above mentioned ingredients were prepared and characterized.

\section{EXPERIMENTAL}

General. NMR spectra were recorded on $400 \mathrm{MHz}$ Bruker FT-NMR spectrometer (Bruker India Scientific Pvt. Ltd., India) using tetramethylsilane as internal standard. Mass spectra were recorded in APCI mode on Finnigan MAT LCQ spectrometer using X'calibur software (Thermo Electron Corp., Waltham, USA) (Provided as Electronic supplementary material).

\section{Synthesis of Ionic Liquids}

IL-1 to $I L-7$. To a solution of 1-methylimidazole $(25 \mathrm{mmol})$ in acetonitrile $(10 \mathrm{~mL})$, was added alkyl bromide (30 mmol) under reflux conditions in presence of acetonitrile. The reaction was allowed to proceed until complete 
Table I. Time Required for Synthesis of Ionic Liquids

\begin{tabular}{|c|c|c|}
\hline Ionic liquid & $\mathrm{R}($ alkyl) & Time (h) \\
\hline IL-1 & $\mathrm{CH}_{3} \mathrm{CH}_{2-}-$ & 0.5 \\
\hline IL-2 & $\mathrm{CH}_{3} \mathrm{CH}_{2} \mathrm{CH}_{2-}$ & 0.5 \\
\hline IL-3 & $\left(\mathrm{CH}_{3}\right)_{2} \mathrm{CH}-$ & 0.5 \\
\hline IL-4 & $\mathrm{CH}_{3} \mathrm{CH}_{2} \mathrm{CH}_{2} \mathrm{CH}_{2-}$ & 1 \\
\hline IL-5 & $\mathrm{CH}_{3} \mathrm{CH}_{2} \mathrm{CH}_{2} \mathrm{CH}_{2} \mathrm{CH}_{2-}$ & 1 \\
\hline IL-6 & $c-\mathrm{C}_{5} \mathrm{H}_{9-}-$ & 1 \\
\hline IL-7 & $\mathrm{CH}_{3} \mathrm{CH}_{2} \mathrm{CH}_{2} \mathrm{CH}_{2} \mathrm{CH}_{2} \mathrm{CH}_{2-}$ & 1 \\
\hline IL-8 & $\mathrm{CH}_{3} \mathrm{CH}_{2-}$ & 3 \\
\hline IL-9 & $\mathrm{CH}_{3} \mathrm{CH}_{2} \mathrm{CH}_{2-}$ & 3.5 \\
\hline IL-10 & $\left(\mathrm{CH}_{3}\right)_{2} \mathrm{CH}-$ & 4.5 \\
\hline IL-11 & $\mathrm{CH}_{3} \mathrm{CH}_{2} \mathrm{CH}_{2} \mathrm{CH}_{2-}$ & 4 \\
\hline IL-12 & $\mathrm{CH}_{3} \mathrm{CH}_{2} \mathrm{CH}_{2} \mathrm{CH}_{2} \mathrm{CH}_{2-}$ & 5 \\
\hline IL-13 & $c-\mathrm{C}_{5} \mathrm{H}_{9-}$ & 6.5 \\
\hline IL-14 & $\mathrm{CH}_{3} \mathrm{CH}_{2} \mathrm{CH}_{2} \mathrm{CH}_{2} \mathrm{CH}_{2} \mathrm{CH}_{2-}$ & 8 \\
\hline
\end{tabular}

conversion of starting material to product as confirmed by thin layer chromatography (TLC). The reaction mixture was washed with diethyl ether, dried under reduced pressure and column chromatography over silica gel (100-200 mesh) was performed to get the quaternized compounds.

$I L-8$ to $I L-12$. Imidazole (25 mmol) was stirred with alkyl bromide $(75 \mathrm{mmol})$ in presence of potassium carbonate $(50 \mathrm{mmol})$ in toluene $(25 \mathrm{~mL})$ under reflux conditions until maximum conversion of starting material to product was observed using TLC. Reaction mixture was dried under vacuo, and column chromatography afforded IL-8 to IL-12.

Table I represents the time required for syntheses of both series of ILs.

\section{In Vitro Hemolysis Assay}

A previously reported method was used with slight modification (13). Briefly, freshly anticoagulated human blood $(5 \mathrm{~mL})$ was diluted with equal volume of phosphate-buffered saline, pH7.4 (PBS). Separately, $0.75 \mathrm{~mL}$ of ILs $(0.75 \mathrm{mg}$ of solid ILs) and solutions of ILs in PBS were incubated in Eppendorf microtubes for $30 \mathrm{~min}$ at $37^{\circ} \mathrm{C}$. Negative and positive controls were prepared by substituting PBS and water, respectively, for the IL (or IL solutions). Diluted blood $(0.25 \mathrm{~mL})$ was added and samples were incubated on a rotator (Innova 42 incubator shaker, New Brunswick Scientific Co. Inc., USA) for $24 \mathrm{~h}$ at $37^{\circ} \mathrm{C}$. The tubes were then centrifuged (10 $\mathrm{min}, 5,000 \mathrm{rpm})$, and the absorbance $(A)$ of the supernatants was measured in a spectrophotometer at $545 \mathrm{~nm}$. The hemolysis degree (HD) was calculated as $100 \% \times\left(A_{\text {sample }}{ }^{-}\right.$ $\left.A_{\text {negative control }}\right) /\left(A_{\text {positive control }}-A_{\text {negative control }}\right)$. Each sample was run in triplicate.

\section{Construction of Pseudoternary Phase Diagrams}

The pseudoternary phase diagrams were constructed at ambient temperature. Mixtures of ILs and Tween 80-Span 80 mixtures (1:1, 1:2 and 1:3 w/w ratios, seperately) were prepared in weight ratios of 0.5:9.5, 1:9, 1.5:8.5, 2:8, 2.5:7.5, 3:7, $3.5: 6.5,4: 6,4.5: 5.5,5: 5,5.5: 4.5,6: 4,6.5: 3.5,7: 3,7.5: 2.5,8: 2$, $8.5: 1.5,9: 1$, and 9.5:0.5. The mixtures showing turbidity were not considered for titrations, and the clear mixtures were titrated drop-wise with IPM with intermediate vortex mixing. After each addition, they were allowed to equilibrate for $5 \mathrm{~min}$ and thereafter visually examined. Transparent fluid systems were characterized as microemulsions, whereas opaque systems were considered as coarse emulsions. No efforts to assess other phases like microgel or emulgel were performed.

\section{Preparation of Microemulsions}

To a thoroughly vortex mixed surfactant mixture (40\%) (Tween 80 and Span 80, 1:3 w/w), ionic liquid (10\%) was added and stirred until homogenous. The required amount of isopropyl myristate $(50 \%)$ was added in small aliquots with vortex mixing. The procedure was carried out at ambient temperature.

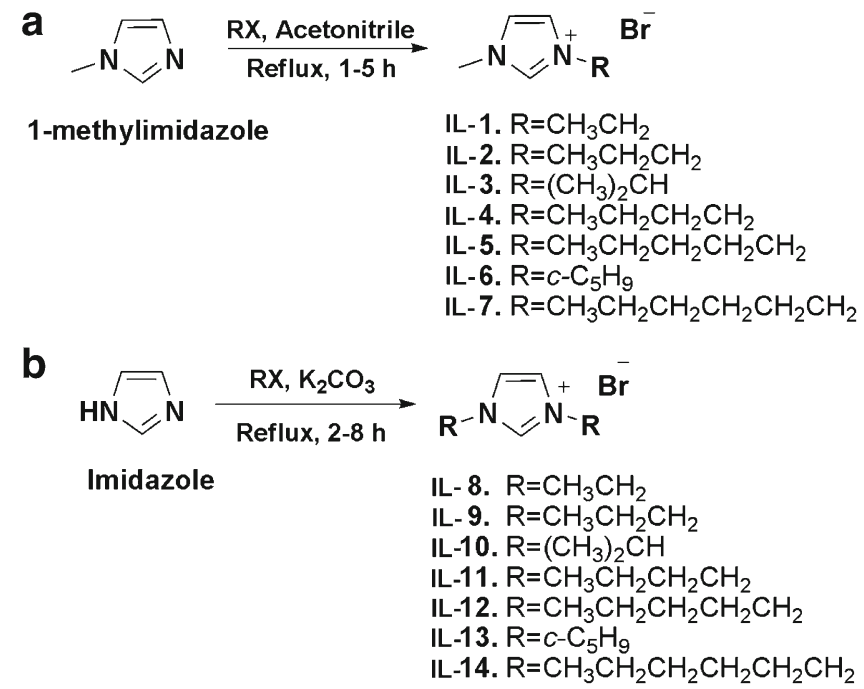

Fig. 1. Synthetic schemes of ionic liquids a IL-1 to IL-7; and b IL-8 and 

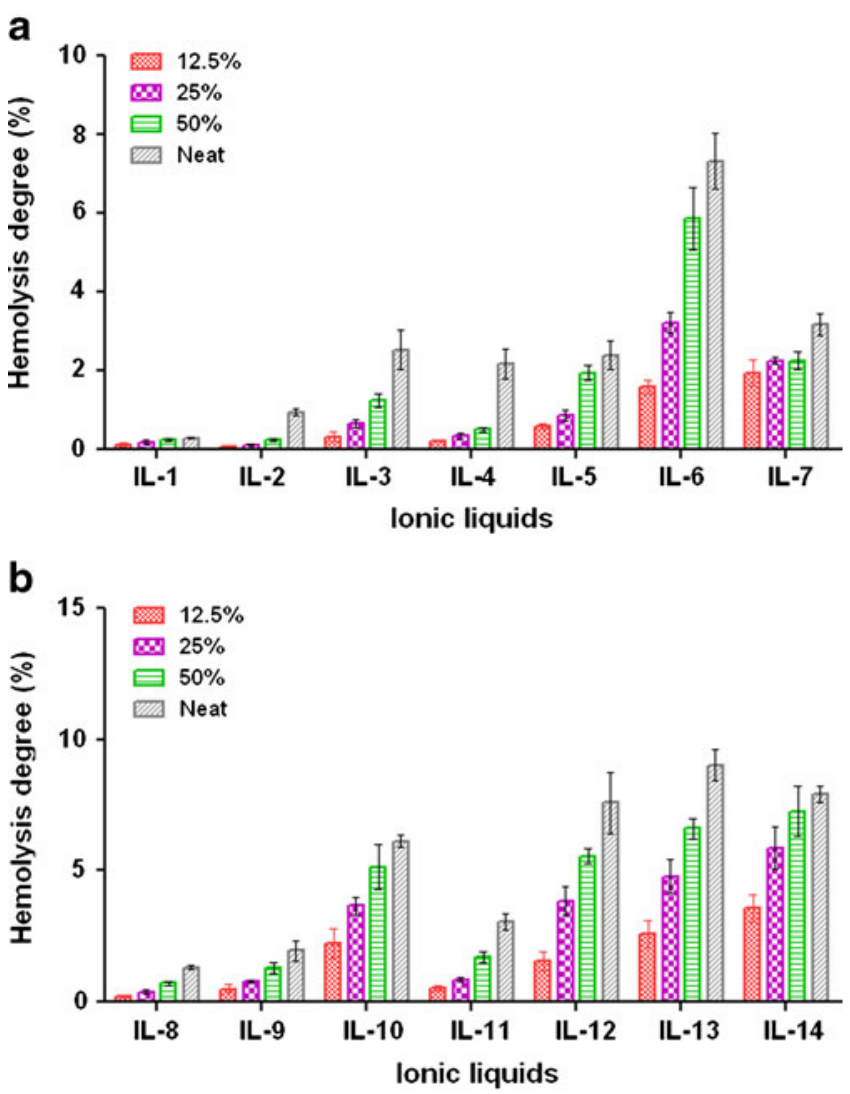

Fig. 2. Hemolysis degree values of a 1-alkyl-3-methylimidazolium bromides (IL-1 to IL-7); and b 1,3-dialkylimidazolium bromides (IL8 to IL-14)

\section{Globule Size and Zeta Potential Determination}

Transmission electron microscopy (H-7500, Hitachi, Japan) was performed after depositing the sample on a filmcoated 200-mesh gold specimen grid. Globule size, polydispersity index, and Zeta potential were determined using Malvern Zetasizer (Malvern Instruments Ltd., UK).

\section{Rheological Profile Study}

The rheological profiles of microemulsions were studied using rotational viscometer (Paar Physica MC1, Brookfield DV-II, UK) equipped with a cup and bob measuring device at $25^{\circ} \mathrm{C}$. The shear stress measurements were performed within the shear rate ranging from 0 to $100 \mathrm{~s}^{-1}$, for both, up and down curves.

\section{pH Value and Conductivity Determination}

The $\mathrm{pH}$ and conductivity values of the microemulsions were determined using Labindia PICO $\mathrm{pH} /$ conductivity meter (Labindia, Mumbai, India).

\section{Thermodynamic Stability Study}

The thermodynamic stability was determined at ambient temperature for 3 months as well as following the three-step procedure as suggested by Shafiq et al. (14) with slight modifications. The microemulsions which were transparent, showed no change in color, and no signs of phase separation were considered to be stable.

1. Heating cooling cycle: Six cycles between refrigerator temperature $\left(4^{\circ} \mathrm{C}\right)$ and $45^{\circ} \mathrm{C}$ with storage at each temperature for $48 \mathrm{~h}$ was studied.

2. Centrifugation: Passed formulations were centrifuged at $10,000 \mathrm{rpm}$ for $30 \mathrm{~min}$.

3. Freeze-thaw cycle: Three freeze-thaw cycles between $-20^{\circ} \mathrm{C}$ and $+25^{\circ} \mathrm{C}$ with storage at each temperature for $48 \mathrm{~h}$ was done for the formulations.

\section{RESULTS AND DISCUSSIONS}

Two series of N-substituted ILs, namely 1-alkyl-3-methylimidazolium and 1,3-dialkylimidazolium cation bromides were prepared (Fig. 1). First series of the ionic liquids was synthesized using single-step quaternization. 1-methylimidazole upon reaction with various alkyl bromides in acetonitrile under reflux conditions provided the compounds IL-1 to IL-7. The second series is composed of ionic liquids with similar alkyl groups attached at the N-1 and N-3 positions. Herein, reaction of imidazole with alkyl bromides in the presence of potassium carbonate yielded compounds IL-8 to IL-14.

The ILs of first series (IL-1 to IL-7) were found to be liquids at room temperature. On the other hand, among the dialkylimidazolium cation-based compounds, 1,3-diethylimidazolium bromide (IL-8) was found to be a viscous semisolid. Also, 1,3-dicyclopentylimidazolium bromide (IL-13) was found to be a pale solid. Similar solid products, while tethering bulky alkyl groups at N-1 and N-3 positions of imidazole ring simultaneously, were reported by some workers in the past $(15,16)$. Although, 1-cyclopentyl-3-methylimidazolium bromide (IL-6) also contains same bulky group as IL-13, it was found to be a liquid. This could be attributed to the fact that apart from the bulky cyclopentyl ring, IL-13 possesses a symmetric imidazolium ring, which is also a major contributing factor for enhancement of melting point of these compounds (17).

The ILs for human use need to be nontoxic. US-FDA recommends in vitro hemolysis assay for testing hemolytic potential of new drugs and excipients (17). This method involves incubating the test compounds with red blood cells (rbc) and then quantifying the percent lysis of rbc using spectrophotometric methods. The absorbance values of negative and positive controls are also assayed, and used to normalize the test absorbance, so that hemolysis can be expressed as a percentage of the positive control (assumed to be $100 \%$

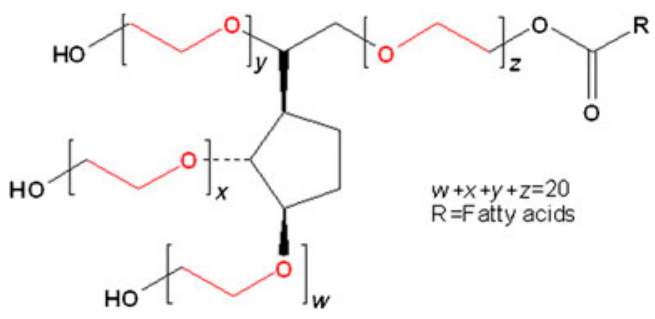

Fig. 3. Structure of polyoxyethylene sorbitan ester (Tween) type surfactants 


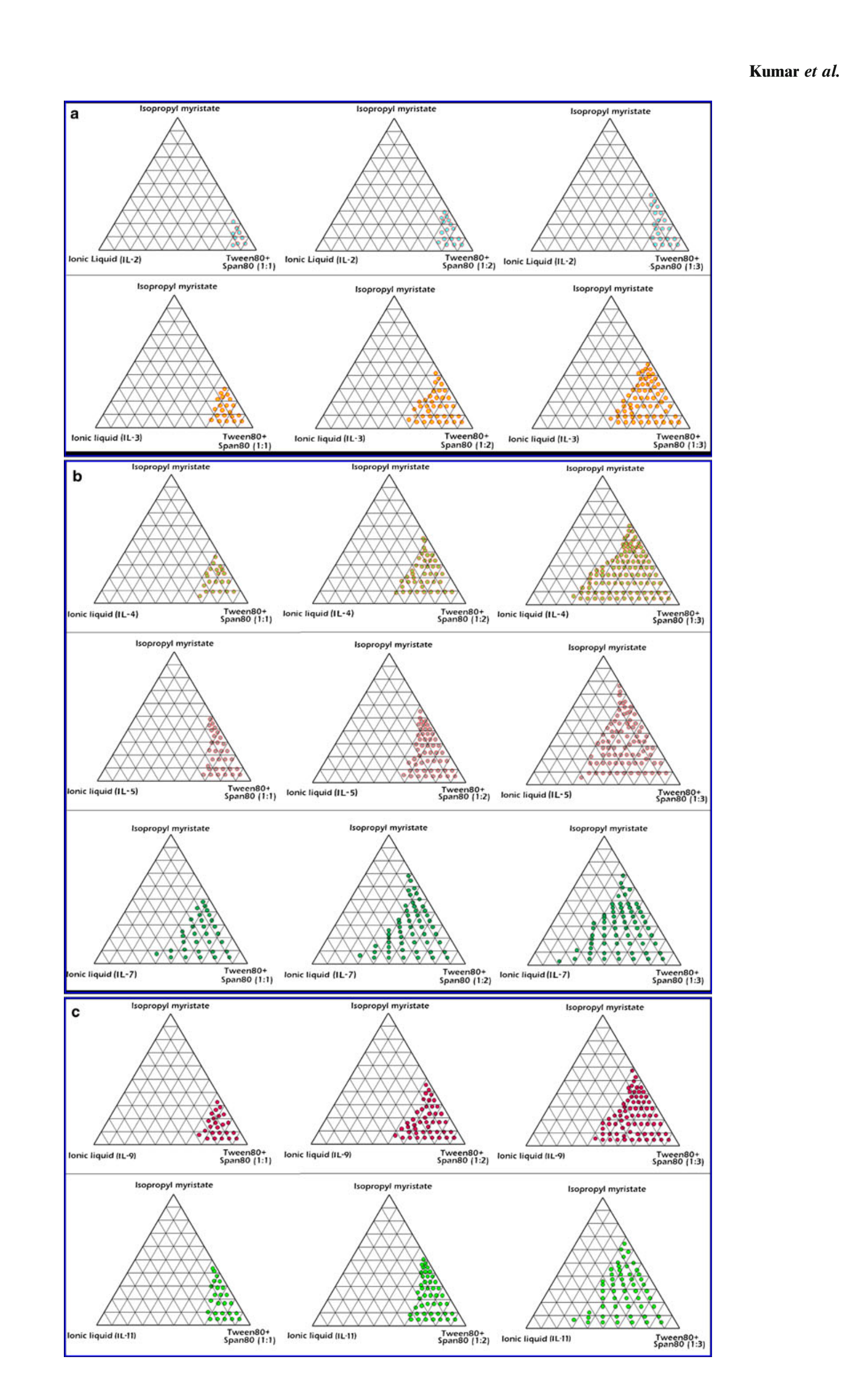


Fig. 4. Pseudoternary phase diagrams prepared with IPM, surfactant mixture and selected ionic liquids. Out of the $3 w / w$ ratios of Tween 80 and Span 80, 1:3 ratio provided best microemulsion existence zones (dotted portions). a IL-2 and IL-3 provided very small microemulsion existence regions; b for IL-4, IL-5 and IL-7, excellent microemulsion existence regions were observed; and c among the 1,3-dialkylimidazolium bromides, IL-11 exhibited better microemulsion-forming ability than IL-9

hemolysis). A sample with hemolysis value less than $5 \%$ is generally considered nonhemolytic. Figure 2 illustrates the results of hemolysis assay for both series of ILs, using them neat and as $50 \%, 25 \%$, and $12.5 \%$ solutions (in phosphatebuffered saline, $\mathrm{pH} 7.4$ ). As per anticipation, the hemolysis was found to be concentration dependant depicting the neat ILs to be most hemolytic and $12.5 \%$ solutions to be least hemolytic for all entries. The 1-alkyl-3-methylimidazolium bromides were clearly less hemolytic than the 1,3-dialkylimidazolium bromides.

It was evident that ILs (of both series) having larger alkyl substituents at N-position were found to be more hemolytic than those having smaller $\mathrm{C}$-chain. For instance, the mean hemolytic degree (HD) values of neat IL-1, IL-2, and IL-4 (with ethyl, propyl, and butyl group, respectively at 1- position of imidazolium ring) were $0.009 \%, 0.086 \%$, and $0.367 \%$, respectively, whereas, those of neat IL-5 and IL-7 (with pentyl and hexyl group, respectively at 1- position of imidazolium ring) were found to be 0.255 and $0.281 \%$, respectively. Similar trend was observed in 1,2-dialkyl imidazolium bromides. Correspondingly, the HD values of neat IL-8, IL-9, and IL11 (with ethyl, propyl and butyl group, respectively at 1- and 3positions of imidazolium ring) were $1.319 \%, 1.949 \%$, and $3.065 \%$, respectively, convincingly lesser than those of IL-12 (7.594\%) and IL-14 (7.917\%).

Also, ILs with branched (isopropyl) substituent were found to be more hemolytic than those having corresponding unbranched alkyl groups. IL-2 and IL-9 (with mean HD values 0.949 and 1.949 , respectively) showed safer hemolytic profiles than IL-3 and IL-10 (with mean HD values of 2.525 and 6.120 , respectively).

In the same way, the ILs having cycloalkyl groups (IL-6 and IL-13) were found to be more toxic than their $n$-alkyl counterparts (IL-5 and IL-12).

Based on their physical state (liquid or flowable nature) and toxicity profile, the representative ILs were selected for pseudoternary phase diagram studies. Therefore, 1,3-diethylimidazolium bromide (IL-7) and 1,3-dicyclopentylimidazolium bromide (IL-13) could not be used due to their solid state at $25^{\circ} \mathrm{C}$. On the other hand, 1-cyclopentyl-3-methylimidazolium bromide (IL-6), 1,3-dipentylimidazolium bromide (IL-12) and 1,3-dihexylimidazolium bromide (IL-14) were rejected due to their greater hemolytic potential than other ILs.

Pseudoternary phase diagram were constructed to identify the microemulsion phase using ILs, oil and emulgent mixture. IPM was used as the oil phase as it is one of the most used pharmaceutical oils and offers certain unique advantages including, non-rancidifying property, odorless and tasteless nature, skin penetration effect (beneficial for dermal drug delivery). For selection of the emulgent phase, different polyoxyethylene sorbitan ester grades (Tween 20, 40, 60, and 80) and sorbitan fatty acid ester grades (Span 20 and 80) surfactants were screened using their mixtures in various ratios $(w / w)$. The mixture of two surfactants was used because in combination they could provide diverse physicochemical properties (18). In addition, the hydrophilic POE groups of Tween surfactants (Fig. 3) are known to have strong affinity for imidazolium cations (19). The selection was made on the basis of the surfactant types capable of solubilizing greatest amount of IPM. Tween 80 and Span 80 mixtures in 1:1,1:2, and 1:3 ratios were found to best in this aspect (data not shown), hence, selected as optimal surfactants to constitute the emulgent phase.

In addition to this, the $1: 1,1: 2$, and $1: 3 w / w$ ratios of these surfactants provide diverse HLB values of 9.65, 7.88, and 6.98, respectively. As illustrated by Moniruzzaman et al. (12), in the microemulsions, the ionic liquid constitutes the hydrophilic internal core, whereas, the surfactant mixtures represents the interfacial film separating the dispersed phase from the oil phase.

1-Ethyl-3-methylimidazolium (IL-1) bromide was found to be insoluble in all ratios of Tween 80 and Span 80 mixtures, hence could not be used for pseudoternary phase diagram studies.

The ternary phase diagrams prepared using selected ILs are represented in Fig. $4 \mathrm{a}-\mathrm{c}$. For all ILs, Tween 80 and Span 80 mixture in 1:3 ratio provided larger microemulsion regions as compared to the other two ratios studied.

It was observed that ILs having longer C-chains showed greater microemulsion region than those having smaller Cchains in pseudoternary phase diagrams. For all surfactant ratios, the microemulsion existence regions in 3-methylimidazolium bromides were found in the order of IL-7 > IL-5 > IL-4 > IL2, while, out of the two 1,3-dialkylimidazolium bromides

Table II. Characteristics of IL/Oil Microemulsions

\begin{tabular}{|c|c|c|c|c|}
\hline Characteristics & ME-1 & ME-2 & ME-3 & ME-4 \\
\hline IL used & IL-4 & IL-5 & IL-7 & IL-11 \\
\hline Globule size (nm) & 77.9 & 137.8 & 178.3 & 101.9 \\
\hline Polydispersity index & 0.262 & 0.276 & 0.233 & 0.185 \\
\hline Viscosity $(\mathrm{cP})$ & 23.5 & 27.6 & 28.9 & 23.5 \\
\hline Rheogram correlation ratio $\left(R^{2}\right)$ & 0.994 & 0.998 & 0.999 & 0.994 \\
\hline Transmittance (\%) & 94.8 & 94.7 & 96.0 & 97.4 \\
\hline $\mathrm{pH}$ & 6.59 & 6.95 & 7.22 & 7.13 \\
\hline Conductivity $(\mu \mathrm{S} / \mathrm{cm})$ & 0.015 & 0.029 & 0.008 & 0.020 \\
\hline Zeta potential (mV) & -0.117 & -0.245 & -0.749 & -0.052 \\
\hline
\end{tabular}




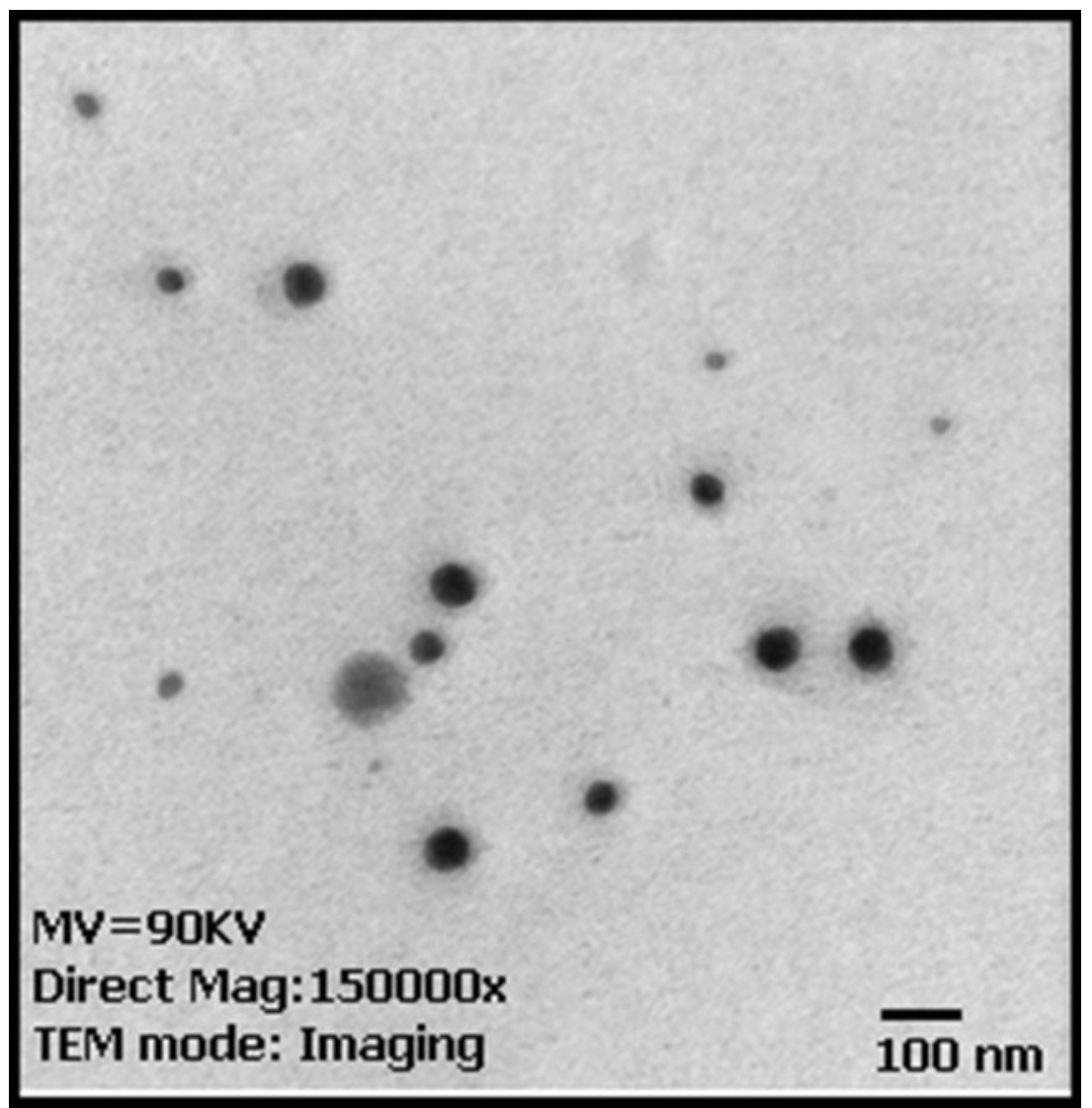

Fig. 5. TEM image of ME-1

studied, IL-11 exhibited greater microemulsion region than IL9. Furthermore, the microemulsion region obtained using 1isopropyl-3-methylimidazolium bromide (IL-3) was greater than 1-propyl-3-methylimidazolium bromide (IL-2).
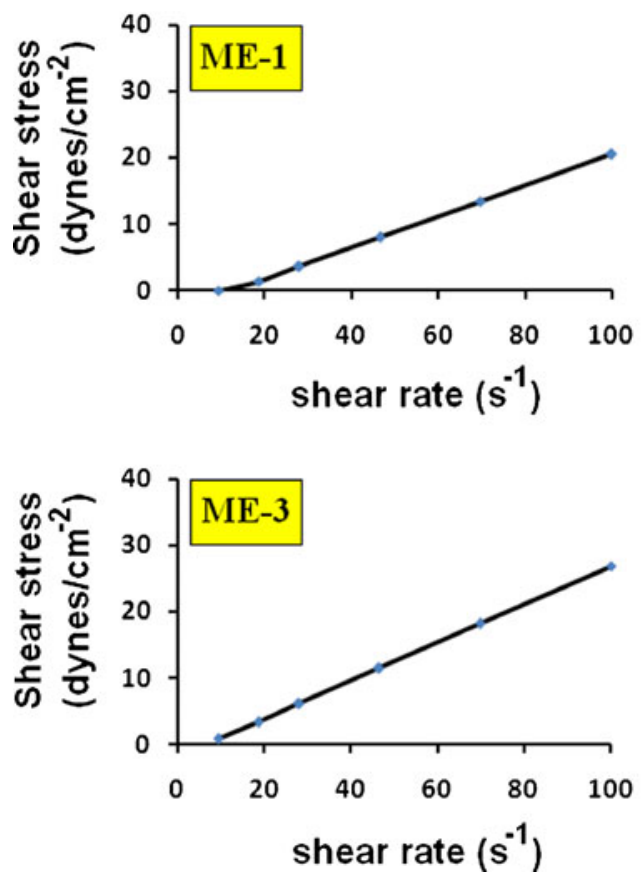

Based on their greater microemulsion regions and lower value of amount of surfactant mixtures required to attain microemulsion phase, IL-4, IL-5, IL-7, and IL-11 could be considered as ILs of choice for preparing
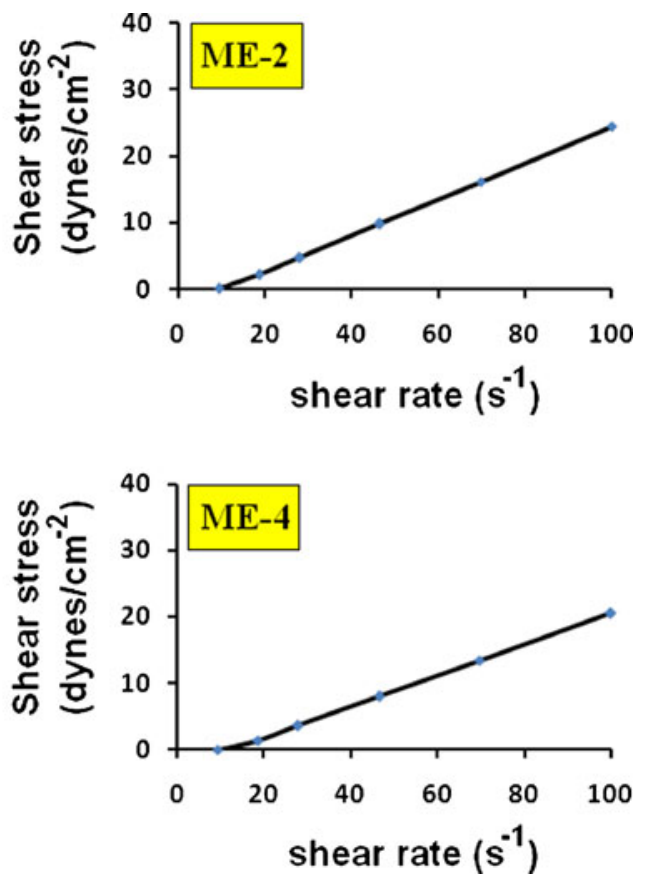

Fig. 6. Rheograms of ME1, ME2, ME3 and ME4 depicting Newtonian flow behavior 
microemulsion formulations. The characteristics of microemulsions prepared using IL-4, IL-5, IL-7, and IL-11 (notated as ME-1, ME-2, ME-3, and ME-4, respectively) are depicted in Table II. The microemulsions showed globule size within $130 \pm 50 \mathrm{~nm}$ range, and low polydispersity index values (depicting monodisperse globule size distribution of microemulsions). Figure 5 depicts TEM image of a representative microemulsion (ME-1), which revealed spherical globules below $100 \mathrm{~nm}$ size range.

Figure 6 represents the rheograms of IL-based microemulsions ME1, ME-2, ME-3, and ME-4. Fluids exhibiting proportionality between shear stress and shear rate are considered to be Newtonian fluids and their viscosity values could be inferred from the slopes of their rheograms. The linear regression $\left(R^{2}\right)$ values of all microemulsions were found to be more than 0.99 , exhibiting their Newtonian flow behavior. This could be attributed to uniformity of size and shape of nanostructures present in the IL/oil microemulsion systems. The microemulsion systems ME-1, ME2, and ME-3 (prepared from ILs within the first series) showed that increase in Cchain length caused slight increase in viscosity values. The $\mathrm{pH}$ values of the microemulsions were found to be similar to skin physiological conditions. The low conductivity values depicted oil-continuous nature of the microemulsions. This implies that the microemulsion systems could be suitable for dermal delivery systems.

The microemulsion systems were found to be stable (in terms of transparency, absence of color change and phase separation) not only after 3 months of storage under ambient conditions, but also when subjected to different thermodynamic stress conditions by using heating cooling cycles, centrifugation, and freeze-thaw cycle tests.

These microemulsions could be used to deliver a variety of poorly soluble and water-labile drugs, which are otherwise difficult to be delivered by w/o microemulsions. Previous studies on IL/O microemulsions report the topical and transdermal applications of these systems $(7,12)$. The microemulsions have been found to enhance in vitro release profile of hydrophobic drugs like acyclovir and progesterone. Therefore, these topical/transdermal microemulsions could provide the hallway for designing new formulations of poorly soluble drugs which are inadequately absorbed by oral route. However, their utility in formulations intended to be used through other routes of drug administration including parental, ocular, and oral use could only be explored after sufficient information on their toxicity profile is obtained.

\section{CONCLUSION}

Two series of imidazolium cation-based ILs having different N-substituents were prepared successfully in good yields. The hemolytic potential of the compounds increased with the C-chain length, and the 3-methylimidazolium cation-based series of ILs was found to be safer than the dialkylimidazolium ILs. The pseudoternary phase diagram studies depicted that an increase in that $\mathrm{C}$ - chain length enhances the microemulsion existence region for the IL in oil microemulsions. IL-4, IL-5, IL-7, and IL-11 were found to be safest and best in terms of greater microemulsion regions, and were used to prepare nanosized clear microemulsions. Although the preliminary results seem promising, more toxicity evaluation parameters need to be studied using different in vivo and in vitro experimental models for establishing these compounds for human use. Our future efforts are directed towards exploring the potential of these ILs in delivery of some hydrophobic drugs.

Declaration of Interest The authors report no declaration of interest.

\section{REFERENCES}

1. Zhou F, Liang Y, Liu W. Ionic liquid lubricants: designed chemistry for engineering applications. Chem Soc Rev. 2009;38:2590-9.

2. Garcia B, Lavallée S, Perron G, Michot C, Armand M. Room temperature molten salts as lithium battery electrolyte. Electrochim Acta. 2004;26:4583-8.

3. Roy SR, Chakraborti AK. Supramolecular assemblies in ionic liquid catalysis for aza-Michael reaction. Org Lett. 2010;12:3866-9.

4. Berthod A, Ruiz-Angel MJ, Carda-Broch S. Ionic liquids in separation techniques. J Chromatogr A. 2008;1184:6-18.

5. Stoimenovski J, MacFarlane DR, Bica K, Rogers RD. Crystalline $v s$. ionic liquid salt forms of active pharmaceutical ingredients: a position paper. Pharm Res. 2010;27:521-6.

6. Pernak J, Sobaszkiewicz K, Mirska I. Anti-microbial activities of ionic liquids. Green Chem. 2003;5:52-6.

7. Jaitely V, Karatas A, Florence AT. Water-immiscible room temperature ionic liquids (RTILs) as drug reservoirs for controlled release. Int J Pharm. 2008;354:168-73.

8. Viau L, Tourné-Péteilh C, Devoisselle J-M, Vioux A. Ionogels as drug delivery system: one-step sol-gel synthesis using imidazolium ibuprofenate ionic liquid. Chem Commun. 2010;46:228-30.

9. Trewyn BG, Giri S, Slowing II, Lin VS-Y. Mesoporous silica nanoparticle based controlled release, drug delivery, and biosensor systems. Chem Commun. 2007:3236-45.

10. Moniruzzaman M, Kamiya N, Goto M. Ionic liquid based microemulsion with pharmaceutically accepted components: formulation and potential applications. J Colloid Interface Sci. 2010;352:136-42.

11. Lawrence MJ, Rees GD. Microemulsion-based media as novel drug delivery systems. Adv Drug Deliv Rev. 2000;45:89-121.

12. Moniruzzaman M, Tamura M, Tahara Y, Kamiya N, Goto M. Ionic liquid-in-oil microemulsion as a potential carrier of sparingly soluble drug: characterization and cytotoxicity evaluation. Int $\mathbf{J}$ Pharm. 2010;400:243-50.

13. Kempe H, Kempe M. The use of magnetite nanoparticles for implant-assisted magnetic drug targeting in thrombolytic therapy. Biomaterials. 2010;31:9499-510.

14. Kumar N, Jain R. Convenient syntheses of bulky group containing imidazolium ionic liquids. J Heterocycl Chem. 2012;49:370-4.

15. Dubey RK, Kumar N, Jain R. Facile syntheses of histamine- and imidazole-4-propionic acid derived room-temperature ionic liquids. Synth Commun. 2012;42:2207-16.

16. Shafiq S, Shakeel F, Talegaonkar S, Ahmad FJ, Khar RK, Ali M. Development and bioavailability assessment of ramipril nanoemulsion formulation. Eur J Pharm Biopharm. 2007;66:227-43.

17. Wasserscheid P, Welton T. Physicochemical properties of ionic liquids. Ionic liquids in synthesis. Weinheim: Wiley-VCH; 2002. p. 49.

18. US Food and Drug Administration. (GUIDANCl5544fnlcln2.doc). Guidance for industry: nonclinical studies for the safety evaluation of pharmaceutical excipients, 2005; pp 1-9.

19. Lu D, Rhodes DG. Mixed composition films of Spans and Tween 80 at the air-water interface. Langmuir. 2000;16:8107-12. 